\title{
The acetyltransferase $60 \mathrm{kDa}$ trans-acting regulatory protein of HIV type 1-interacting protein (Tip60) interacts with the translocation E26 transforming-specific leukaemia gene (TEL) and functions as a transcriptional co-repressor
}

\author{
Iver NORDENTOFT and Poul JøRGENSEN ${ }^{1}$ \\ Department of Molecular Biology, Aarhus University, C. F. Møllers Allé 130, DK 8000 Aarhus C, Denmark
}

The translocation E26 transforming-specific (ETS) leukaemia (TEL), alias the ETS variant (ETV6), gene is expressed in most human tissues and encodes a transcriptional repressor. The TEL gene is involved in more than 40 different chromosomal translocations associated with haematological malignancies. As little is known about the function of intact TEL, we searched for TEL-interacting proteins by yeast two-hybrid screening. Among the interacting partners, we identified the histone acetyltransferase protein Tip60 [60 kDa trans-acting regulatory protein of HIV type 1 (Tat)-interacting protein]. The interaction was reproduced in vitro, and in mammalian cells we mapped the interaction regions in TEL to the ETS domain and those in Tip60 to the MYST (' $\underline{\mathrm{MOZ}}, \underline{\mathrm{Y}} b f 2 / \mathrm{Sas} 3, \underline{\mathrm{S} A S 2}$ and Tip60', where MOZ stands for male absent on the first, SAS for something about silencing and Ybf2 for identical with SAS2) region. Detailed analysis of the Tip60 MYST domain by introduction of point mutations revealed that an $\mathrm{N}$-terminal $\mathrm{C}_{2} \mathrm{HC}$ zinc finger was essential for interaction with TEL. Finally, we showed that Tip60 functions in a reporter system as a co-repressor in TEL-mediated transcription repression.

Key words: E26 transforming-specific transcription factor, histone acetyltransferase, translocation E26 transforming-specific leukaemia (TEL), $60 \mathrm{kDa}$ trans-acting regulatory protein of $\mathrm{HIV}$ type 1-interacting protein (Tip60), transcription.

\section{INTRODUCTION}

The translocation E26 transforming-specific (ETS) leukaemia (TEL) gene was identified originally as a fusion partner to the platelet-derived growth factor receptor $\beta$ at $\mathrm{t}(5 ; 12)(\mathrm{q} 33 ; \mathrm{p} 13)$ chromosomal translocation associated with chronic myelomonocytic leukaemia [1] and was subsequently denoted the ETS variant (ETV6) gene. TEL is expressed in most of the human and mouse tissues [2,3], and is essential during embryonic development since inactivation in mice by knock-out techniques causes severe defects in the developing vascular network of yolk sac and the embryo dies at day E10.5-11.5 [3]. TEL is disrupted in a large number of chromosomal translocations involving various tyrosine kinases \{Abelson mouse leukaemia viral oncogene homologue ABL(9q34) [4], Abelson-related gene ARG(1p13) [5], Janus kinase JAK2(9p24) [6]\}, tyrosine kinase receptor C TRKC(15q24) [7] and transcription factors \{acute myeloid leukaemia (AML) 1/Runt-related transcription factor 1 (21q22) [810], ecotropic viral integration site EVI1(3q26) [11] and caudaltype homeobox transcription factor CDX2(13q12) [12]\}. TEL contains two functional domains: (i) the $\mathrm{N}$-terminal pointed (PNT) homodimerization domain that is homologous with a region in the Drosophila protein pointed [1] and (ii) a C-terminal ETS DNA-binding domain (DBD) [2,13]. In translocations involving the tyrosine kinases, the TEL 5'-region including the PNT domain is fused to the tyrosine kinases, and PNT facili- tates dimerization of the fusion protein, leading to constitutive activation of tyrosine kinase. In other translocations (TEL/ ecotropic viral integration site EVI1 and TEL/caudal-type homeobox transcription factor CDX2), the contribution of TEL is believed to be as an active promoter, because no functional TEL domains are included in the fusion protein. In the most frequent TEL translocation $[\mathrm{t}(12 ; 21)(\mathrm{p} 13 ; \mathrm{q} 22)]$, the TEL Nterminal region (residues 1-336) including PNT is fused to the AML1 DBD and transactivation domains (residues 21480). This translocation is associated with the most common paediatric B-cell acute lymphoblastic leukaemia (ALL) [14]. The non-translocated TEL allele is deleted frequently in the TEL/AML1 type of ALL, suggesting a role for TEL as a tumour suppressor $[9,10,15]$, and is supported by two observations: (i) TEL expression reverses Ras-induced transformation of NIH3T3 cells $[16,17]$ and (ii) TEL inhibits the expression of the metalloproteinase stromelysin-1 by directly binding to the promoter, which is important in tissue remodelling and tumour cell invasion. TEL functions as a repressor of transcription [18] and the repression is associated with the binding of the corepressors $\mathrm{mSin} 3 \mathrm{~A}$ and silencing mediator for retinoid and thyroid receptor/nuclear co-repressor [19-21].

Tip60 [60 kDa trans-acting regulatory protein of HIV type 1 (Tat)-interacting protein] was identified as an interaction partner for the HIV-1 Tat protein and was shown to increase Tat transactivation of the HIV-1 promoter [22]. Tip60 has a chromatin

Abbreviations used: ALL, acute lymphoblastic leukaemia; AML1, acute myeloid leukaemia 1; AR, androgen receptor; CHROMO, chromatin organization modifier; DBD, DNA-binding domain; EBS, ETS-binding sites; Esa1, essential sas family acetyltransferase 1; ER, oestrogen receptor; ERE, oestrogen response element; ETS, E26 transforming-specific (domain); GST, glutathione S-transferase; HAT, histone acetyltransferase; MOZ, male absent on the first; SAS, something about silencing; Tat, trans-acting regulatory protein of HIV type 1; Tip60, 60 kDa Tat-interacting protein; Ybf2, identical with SAS2; MYST, 'MOZ, Ybf2/Sas3, SAS2 and Tip60'; NR, nuclear receptor; PNT, pointed domain; SEAP, secreted alkaline phosphatase; TEL, translocation ETS leukaemia (gene); VP16, virus protein 16; X-Gal, 5-bromo-4-chloroindol-3-yl $\beta$-D-galactopyranoside.

1 To whom correspondence should be addressed (email pj@mbio.aau.dk). 
organization modifier (CHROMO) domain in its N-terminal end and a MYST domain named after its founding members (' $\underline{M O Z}, \underline{Y} b f 2 / S a s 3, \underline{S A S} 2$ and Tip60', where MOZ stands for male absent on the first, SAS for something about silencing and Ybf2 is identical with SAS2) in the C-terminal end. The MYST domain defines Tip60 as belonging to the MYST family of histone acetyltransferase (HAT) proteins that are conserved from yeast to human (see [23] for a review). HATs are associated mostly with transcription activation and function as catalytic subunits of large protein complexes. The model is that the HATs acetylate the basic histone tails, leading to a chromatin structure more accessible for regulatory proteins. The acetylated lysine residues have been proposed to form a protein-binding site for protein containing bromodomains [24,25]. Purified Tip60 acetylates the individual histones $\mathrm{H} 4, \mathrm{H} 3$ and $\mathrm{H} 2 \mathrm{~A}$, whereas histones in nucleosome particles are acetylated poorly [26,27]. Tip60 is identified as a HAT subunit of a large HAT complex [28], which is most probably the mammalian homologue of the yeast nucleosomal acetyltransferase $(\mathrm{NuA} 4)$ complex, which includes the essential sas family acetyltransferase (Esa) 1, supposed to be the functional equivalent of Tip60 [29]. The Tip60 complex readily acetylates nucleosome-assembled histones $\mathrm{H} 4$ and H2A. Besides Tip60, the complex also contains two proteins, TAP54 $\alpha /$ Tip49a and TAP54 $\beta /$ Tip49b (where TAP stands for Tip60-associated protein and Tip49 the $49 \mathrm{kDa}$ TATA-boxbinding protein (TBP)-interacting protein), which are related to the Escherichia coli RuvB hexameric ring helicase protein that plays a role in the repair of damaged DNA. Supporting this finding, the HAT domain of Tip60 is shown to be required for the effective DNA repair of double-strand breaks and for apoptosis after $\gamma$-irradiation of human cells [28]. Moreover, Tip60 binds to the $\beta$-amyloid precursor protein (APP) and enhances apoptosis [30]. Recently, it has been shown that the ubiquitin ligase mouse double minute clone 2 oncoprotein targets Tip60 for degradation, in a manner similar to mouse double minute clone 2 oncoproteinmediated degradation of p53 [31]. Finally, Tip60 functions as a co-activator of class I nuclear hormone receptors [32-34].

In the present study, we report that TEL and Tip60 interact in vitro and in vivo and show that Tip60 in a reporter system functions as a co-repressor for TEL in the regulation of transcription.

\section{MATERIALS AND METHODS}

\section{Yeast two-hybrid screening}

Full-length TEL (bait) was fused to the Lex A DBD (amino acids 1-202) in pBTM116, creating pBTM116-TEL. Yeast

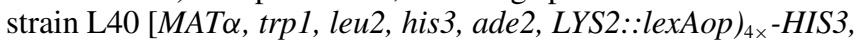
URA3::(lexAop $)_{8 \times}$-LacZ] was transformed sequentially with pBTM116-TEL and the human leucocyte library (ClonTech Laboratories, Palo Alto, CA, U.S.A.) in accordance with the manufacturer's instructions. The cDNA library is positioned in the prey vector $\mathrm{pACT} 2$, where Gal4 AD (768-881 amino acids) form the $\mathrm{N}$-terminal part of the fusion. We screened $1.9 \times 10^{6}$ clones. L40 contains histidine and LacZ reporter genes. Positive clones were selected by prototrophic growth on solid media without histidine, but with $5 \mathrm{mM} 3$-amino-1,2,3 triazole. Activation of the LacZ gene was verified using $\beta$-galactosidase overlay assay: L40 transformants containing the bait-LexA (pBTM116) plasmid and prey-Gal4 AD (pACT2) were grown for 2-3 days on SC-Trp, Leu or SC-Trp, Leu and His plates. Then, $0.5 \%$ low-melting agarose in $0.5 \mathrm{M}$ potassium phosphate buffer ( $\mathrm{pH} 7.0$ ) was prepared and cooled to $60{ }^{\circ} \mathrm{C}$, then $0.1 \%$ (v/v) SDS, $6 \%$ (v/v) 1-methyl2-pyrrolidone and $0.05 \%$ (w/v) 5-bromo-4-chloroindol-3-yl $\beta$-D-galactopyranoside (X-Gal) were added. The plates were then carefully overlaid with agarose. When solidified, the plates were inverted and incubated at $30^{\circ} \mathrm{C}$. Activation of LacZ will hydrolyse $\mathrm{X}-\mathrm{Gal}$ and will be visualized as a blue colour within a few hours. Prey plasmids from positive clones were purified and cloned into E. coli MC1066 [trpC9830, leuB600, pyrF:: Tn5 lac $\Delta X 74$, strA, galU, $\left.\operatorname{galK}\left(r^{-} \mathrm{m}^{+}\right)\right]$and plated on to M9 medium without leucine. The pACT2 prey plasmid was purified and sequenced.

\section{Interaction mapping in yeast}

TEL deletions were fused to the LexA DBD (1-202 amino acids) by cloning into pBTM116. Tip60 deletions were fused to Gal4 AD (768-881 amino acids) by cloning into pACT2 (ClonTech Laboratories). All constructs were cloned by PCR using PFU turbo DNA polymerase (Stratagene, La Jolla, CA, U.S.A.) and verified by sequence determination (DYEnamic ET Terminator Cycle Sequencing premix kit; Amersham Biosciences, Uppsala, Sweden). Sequences were detected on an ABI Prism 3100 Genetic Analyzer capillary machine (AB Applied Biosystems, Foster City, CA, U.S.A.). Interactions were monitored as described for the yeast two-hybrid screen.

\section{Plasmids}

pACT2-Tip60 $\alpha$ and the corresponding deletions were PCRcloned from IMAGE clone 417933. The pACT-Tip60 $\beta$ was isolated in the yeast two-hybrid screening and deletions were derived by PCR-aided cloning. The secreted alkaline phosphatase (SEAP) reporter construct directs transcription of a SEAP gene under the control of a $\beta$-globin promoter [35]. An oestrogen response element (ERE) and two Gal4-binding sites are positioned upstream of the promoter. The reporter EBS-SEAP (where EBS represents ETS-binding sites) is constructed by inserting three TEL EBS between the Gal4 and ERE sites. The sequence ATAAACAGGAAGTGGTTCGGAAGTGAGGAAGTGAATTC was introduced using the QuickChange Site-Directed Mutagenesis kit (Stratagene) in accordance with the manufacturer's instructions.

\section{Interactions in vitro}

His-tagged TEL was obtained by cloning TEL into pRSET B (Invitrogen, Carlsbad, CA, U.S.A.) and expressed in E. coli BL21(DE3)pRI952. His-TEL was affinity-purified using $\mathrm{Ni}^{2+}$ nitrilotriacetate beads (Amersham Biosciences) in accordance with the manufacturer's instructions. Glutathione S-transferase (GST) fusions of Tip60 $\beta$ and Tip60 $\alpha$ (212-513) were cloned into pGEX-5x-2 (Amersham Biosciences) and expressed in E. coli BL21 (DE3) pRI952; $5 \mu \mathrm{g}$ of GST-Tip60 $\beta$ and GST-Tip60 $\alpha$ (212-513), and 20 or $2 \mu \mathrm{g}$ of GST (control) were immobilized on glutathione-Sepharose $4 \mathrm{~B}$ beads ( $60 \mu \mathrm{g}$ of bed volume) by incubation for $2 \mathrm{~h}$ under rotation at $4{ }^{\circ} \mathrm{C}$ in $1 \mathrm{ml}$ of NETN buffer [2 mM EDTA, $100 \mathrm{mM} \mathrm{NaCl}, 25 \mathrm{mM}$ Tris (pH 8), $0.5 \%$ Nonidet P40, $1 \mathrm{mM}$ PMSF and $1 \times$ complete proteinase inhibitor cocktail (Roche, Mannheim, GmbH, Germany)]. The beads were washed five times in NETN buffer and $0.5 \mu \mathrm{g}$ of affinity-purified His $_{6 \times}-$ TEL was added and incubated in $1 \mathrm{ml}$ of NETN (1 mM PMSF and $1 \times$ complete proteinase inhibitor cocktail) overnight to allow binding. The beads were again washed five times in NETN. Finally, bound protein was eluted by boiling in denaturing SDS-loading buffer (50 mM dithiothreitol) and resolved on SDS/ PAGE, followed by Western blotting and antibody probing with primary anti-His rabbit polyclonal IgG (DAKO, Glostrup, Denmark) and secondary horseradish peroxidase-conjugated rabbit immunoglobulins (DAKO). 


\section{Mammalian two-hybrid analysis}

Gal4-tagged TEL constructs were obtained by cloning into the expression vector pM (pPC97; ClonTech Laboratories) modified with a new multiple cloning site [35]. Virus protein 16 (VP16)tagged Tip60 constructs were obtained by cloning into pTLI-VP16 (A.L. Nielsen, personal communication). The SEAP reporter construct directs transcription of a SEAP gene under the control of a $\beta$-globin promoter.

\section{Cells and transfections}

Culturing of HT1080 cells was performed in Dulbecco's modified Eagle's medium, containing glutamax, $10 \%(\mathrm{v} / \mathrm{v})$ foetal calf serum and $2 \%(\mathrm{v} / \mathrm{v})$ penicillin-streptomycin. HT1080 cells at $70 \%$ confluence were transfected using Superfect (Qiagen, Hilden, $\mathrm{GmbH}$, Germany) according to the manufacturer's instructions using $1 \mu \mathrm{g}$ of Gal4-ERE-SEAP reporter vector and $1 \mu \mathrm{g}$ of pCH110 $\beta$-gal internal control vector. Transfections were performed in triplicate in $60 \mathrm{~mm}$ dishes. For the repression assay (Figure 6), TEL and Tip60 were expressed from pCMV (ClonTech Laboratories).

\section{Enzyme assays}

For SEAP assays, medium samples of $200 \mu \mathrm{g}$ were taken 24 and $48 \mathrm{~h}$ after transfection. The samples were heated at $65^{\circ} \mathrm{C}$ for 10 $15 \mathrm{~min}$. Heat incubation was done to inactivate wild-type alkaline phosphatases. The SEAP reporter protein was thermostable. Then $150 \mu \mathrm{g}$ of SEAP substrate (Sigma-Aldrich, St. Louis, MO, U.S.A.) was added to each sample and mixed. The plate was incubated at $37{ }^{\circ} \mathrm{C}$ and $A_{405}$ measured at different time intervals for a $24 \mathrm{~h}$ period. SEAP activity was calculated as follows:

$$
\begin{aligned}
\mathrm{SEAP} \text { activity } / \mathrm{h}= & \left\{\left(\Delta A_{405} / \text { time }\right)_{48 \mathrm{~h} \mathrm{sample}}\right. \\
& \left.-\left[\Delta A_{405} / \text { time }(\text { media })\right]_{48 \mathrm{~h} \mathrm{sample}}\right\} \\
& -\left\{\left(\Delta A_{405} / \text { time }\right)_{24 \mathrm{~h} \mathrm{sample}}\right. \\
& \left.-\left[\Delta A_{405} / \text { time }(\text { media })\right]_{24 \mathrm{~h} \mathrm{sample}}\right\}
\end{aligned}
$$

SEAP values were normalized to $\beta$-galactosidase values.

For $\beta$-galactosidase assays, media were removed from the 6well dishes and the cells were washed in $1 \mathrm{ml}$ of PBS. Trypsin $(200 \mu \mathrm{l})$ was added to each well and the plate was incubated for $5 \mathrm{~min}$ at $20^{\circ} \mathrm{C}$. Then, $1 \mathrm{ml}$ of PBS was added and pipetted up and down to remove all cells from the disc surface, before transferring them to Eppendorf tubes. The cells were resuspended in $100 \mu \mathrm{l}$ of $250 \mathrm{mM}$ Tris ( $\mathrm{pH} \mathrm{7.8)}$ by vortex-mixing and then lysed by freezing in liquid nitrogen for 3 min and thawing in a water bath. This was repeated five times intervened by vigorous vortex-mixing. Finally, the tubes were centrifuged for $10 \mathrm{~min}$ at $10000 \mathrm{rev} . / \mathrm{min}$ and $10 \mu \mathrm{l}$ of supernatant/cell extract was mixed with $65 \mu \mathrm{l}$ of $4 \mathrm{mg} / \mathrm{ml} o$-nitrophenyl $\beta$-D-galactopyranoside. The plate was incubated at $37{ }^{\circ} \mathrm{C}$ and $A_{405}$ measured at different time intervals. The transformation efficiency was then calculated as $\Delta A_{405}-\Delta A_{405}$ (media)/time unit.

\section{RESULTS}

\section{$\operatorname{Tip} 60 \beta$ is identified as an interaction partner to TEL}

To illuminate the function of TEL, we searched for protein interaction partners using the yeast two-hybrid system [36]. Briefly, the yeast strain L40 was transfected sequentially with fulllength TEL fused to LexA (bait) and a human leucocyte cDNA

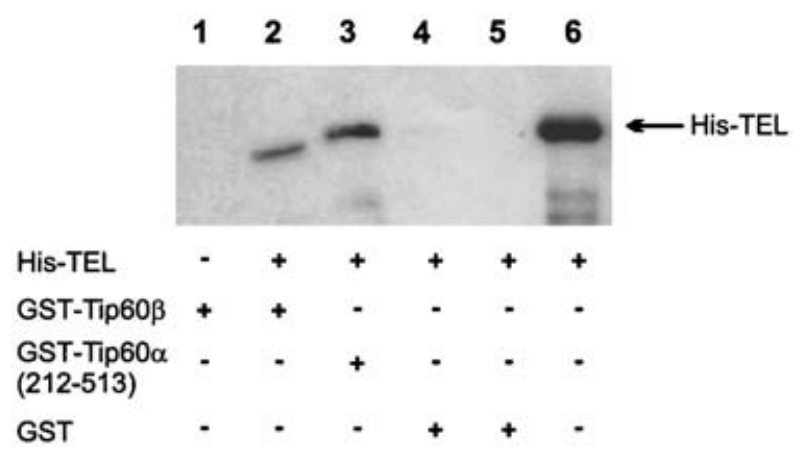

Figure 1 Association of $\mathrm{His}_{6 \mathrm{x}}-\mathrm{TEL}$ with GST-Tip60 $\beta$ and GST-Tip60 $\alpha$ (212-513) in vitro

GST-Tip60 $\beta$, GST-Tip60 $\alpha$ (212-513) and GST expressed in BL21 (DE3) pRI952 were immobilized on glutathione-Sepharose beads, then incubated with $\mathrm{Ni}^{2+}$-nitrilotriacetate affinitypurified His 6x $_{x}$-TEL [expressed in BL21 (DE3) pRI952], washed and eluted. The pull-down reactions were resolved by SDS/PAGE $[4 \%(\mathrm{w} / \mathrm{v}) / 15 \%(\mathrm{w} / \mathrm{v})]$ and analysed by Western blotting and probed with primary anti-His rabbit polyclonal IgG and secondary horseradish peroxidaseconjugated rabbit immunoglobulins. The gels were loaded as follows: lane 1, GST-Tip60 $\beta$ $\left(5 \mu \mathrm{g}\right.$ ), negative control; lane 2, GST-Tip60 $\beta$ (approx. $5 \mu \mathrm{g}$ ) + His sx $_{6 x}-$ TEL (approx. $0.5 \mu \mathrm{g}$ ); lane 3, GST-Tip60 $\alpha$ (212-513) (approx. $5 \mu \mathrm{g})+$ His $_{6 x}-$ TEL (approx. $\left.0.5 \mu \mathrm{g}\right)$; lane 4, GST (approx. $20 \mu \mathrm{g})+\mathrm{His}_{6 \times}-\mathrm{TEL}($ approx. $0.5 \mu \mathrm{g}$ ); lane 5, 1/10 GST (approx. $2 \mu \mathrm{g})+\mathrm{His}_{6_{x}}-$ TEL (approx. $0.5 \mu \mathrm{g}$ ); lane 6, $20 \%$ of input His s. $_{x}$-TEL (approx. $0.1 \mu \mathrm{g}$ ).

library (prey). We screened $1.9 \times 10^{6}$ transformants and identified 62 clones that were positive for the expression of the selection markers, histidine residue and $\beta$-galactosidase. Sequence analysis showed that more than $50 \%$ of the clones were coding for the human small ubiquitin-related modifier-1/ubiquitin-conjugating enzyme UBC9, previously confirmed to have affinity to TEL and mediate TEL sumoylation [37]. Among the residual interacting clones, we found protein inhibitor of signal transducer and activator of transcription 1 (PIAS1) [38], Flice-associated huge protein (FLASH) [39] and death-associated protein (DAXX) [40,41], suggested to be involved in degradation and apoptosis; zinc finger proteins ZNF198 [42] and ZNF237 [43], which are putative transcription factors; peptidyl-prolyl cis/trans-isomerase, Nima-interacting (PIN1), which is a peptidyl-prolyl cis/transisomerase (PPIase) of unknown function [44,45]; and finally a clone of interest for the function of TEL as a transcription regulator, the Tip60 protein, which is a HAT. We identified Tip60 in two independent clones that encoded the $\mathrm{C}$-terminal section and full-length Tip60 $\beta$ respectively. The C-terminal section is common to the Tip60 $\alpha$ and Tip60 $\beta$ splice versions (see Figure 3 ). To analyse for differences between the two Tip60 splice variants, both were cloned and tested in yeast. Tip60 $\alpha$ was similar to Tip60 $\beta$ in binding TEL, although the interaction seemed a little weaker when estimated from LacZ activation on plates (results not shown). The interaction between TEL and Tip60 is specific, since co-transformation of Tip60 and the empty bait vector or bait vector encoding lamin does not activate the selection markers.

\section{TEL interacts directly with Tip60 in vitro}

To show that the interaction between Tip60 and TEL was direct and not assisted by proteins from yeast, we performed a pulldown assay in vitro using proteins produced in E. coli. Tip60 $\beta$ and the N-terminal deletion Tip60 $\alpha$ (212-513), both fused to GST and His-tagged TEL, were expressed in E. coli BL21 (DE3) pRI952 and used for GST pull-down assays (see Figure 1). Both Tip60 $\beta$ and Tip60 $\alpha$ (212-513) bound to TEL (lanes 2 and 3), whereas no binding was detected to GST alone (lanes 4 and 5). The interaction analyses were performed under standard conditions 


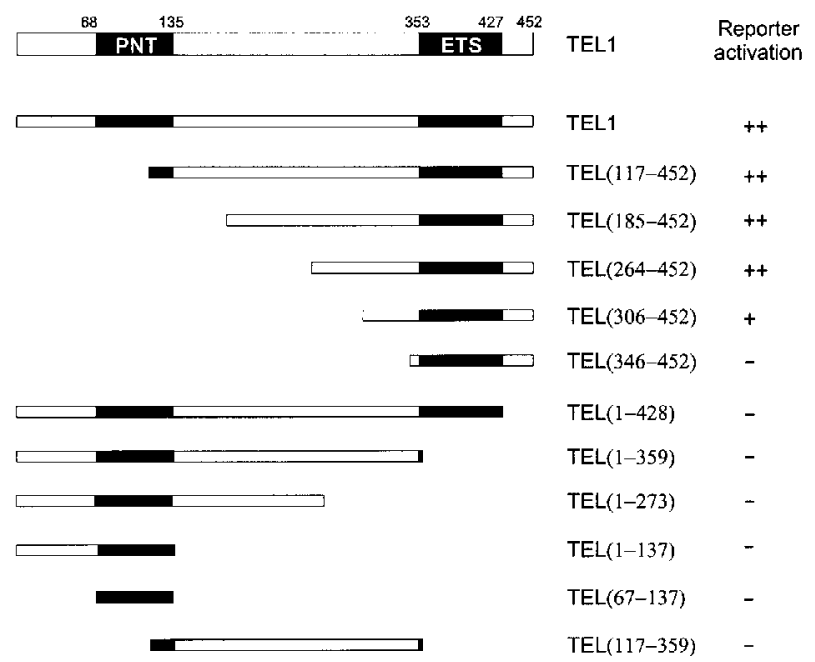

Figure 2 Tip60 interaction region in TEL

Tip60 $\alpha$ and Tip60 $\beta$ fused to the Gal4 (amino acids 768-881) activation domain and TEL deletions fused to the DBD LexA (amino acids 1-202) were tested for interaction in the yeast two-hybrid system using the yeast strain L40, containing the HIS3 and LacZ reporter genes. Interactions were detected as prototrophic growth on solid media lacking histidine and blue colouring when grown in the presence of X-Gal (see the Materials and methods section). The TEL interaction with Tip60 $\alpha$ and Tip60 $\beta$ gave identical results. The functional expression of the different TEL deletions was verified in the yeast two-hybrid system using the other interaction partners that we found in the primary screening (protein inhibitor of signal transduction and activators of transcription (PIRS1), Flice-associated huge protein (FLASH) and death-associated protein (DAXX); results not shown). The TEL ETS domain with flanking regions (amino acids 264-345 and 429-452) are required for Tip60 $\alpha$ and Tip60 $\beta$ interaction with TEL.

at $100 \mathrm{mM} \mathrm{NaCl}$. Furthermore, the His antibody did not detect Tip60 $\beta$ or any degradation product (lane 1). In conclusion, results confirmed direct interaction between Tip60 and TEL.

\section{The C-terminal third of TEL mediates interaction with Tip60}

To map the region of TEL responsible for Tip60 binding, fragments of TEL were tested against full-length Tip60 $\alpha$ and Tip60 $\beta$ in the yeast two-hybrid system (Figure 2). Deletion of 263 N-terminal amino acids of TEL [clone TEL (264-452)] did not affect the interaction with Tip60. However, removing 42 additional amino acids [clone TEL (306-452)] weakened the Tip60 interaction significantly and the deletion of further 82 amino acids [clone TEL (346-452)] eliminated all interactions. Deletion of only 24 amino acids from the C-terminal, leaving the ETS domain intact [clone TEL (1-428)], discontinued all interactions with Tip60. Results suggest that the ETS domain in TEL is responsible for the interaction with Tip60 and that the flanking regions influence the interaction.

\section{MYST domain in Tip60 mediates TEL binding}

The TEL interaction region in Tip60 was mapped by constructing Tip60 deletion mutants that were tested for interaction with TEL in the yeast two-hybrid system (Figure 3). Deletion of the $34 \mathrm{C}$-terminal amino acids [creating clones Tip60 $\alpha(1-479)$ and Tip60 $\beta$ (1-427)] eliminated interaction with TEL. This extreme C-terminal region contains the $\mathrm{C}$-terminal part of the MYST domain showing least conservation compared with the closely homologous MYST domains of human MOZ, MOZ-related factor (MORF) and HBO1. Furthermore, this region contains a nuclear receptor box (NR-box); LXXLL is normally present in nuclear hormone receptor-binding proteins. Although NRbinding proteins usually apply more than one NR-box for binding, Tip60 is shown to function as a co-activator for class I NR by directly binding to the Tip60 NR-box, the function being dependent on the functional HAT activity of Tip60 [32-34]. Analysis of the N-terminal deletions showed that the first

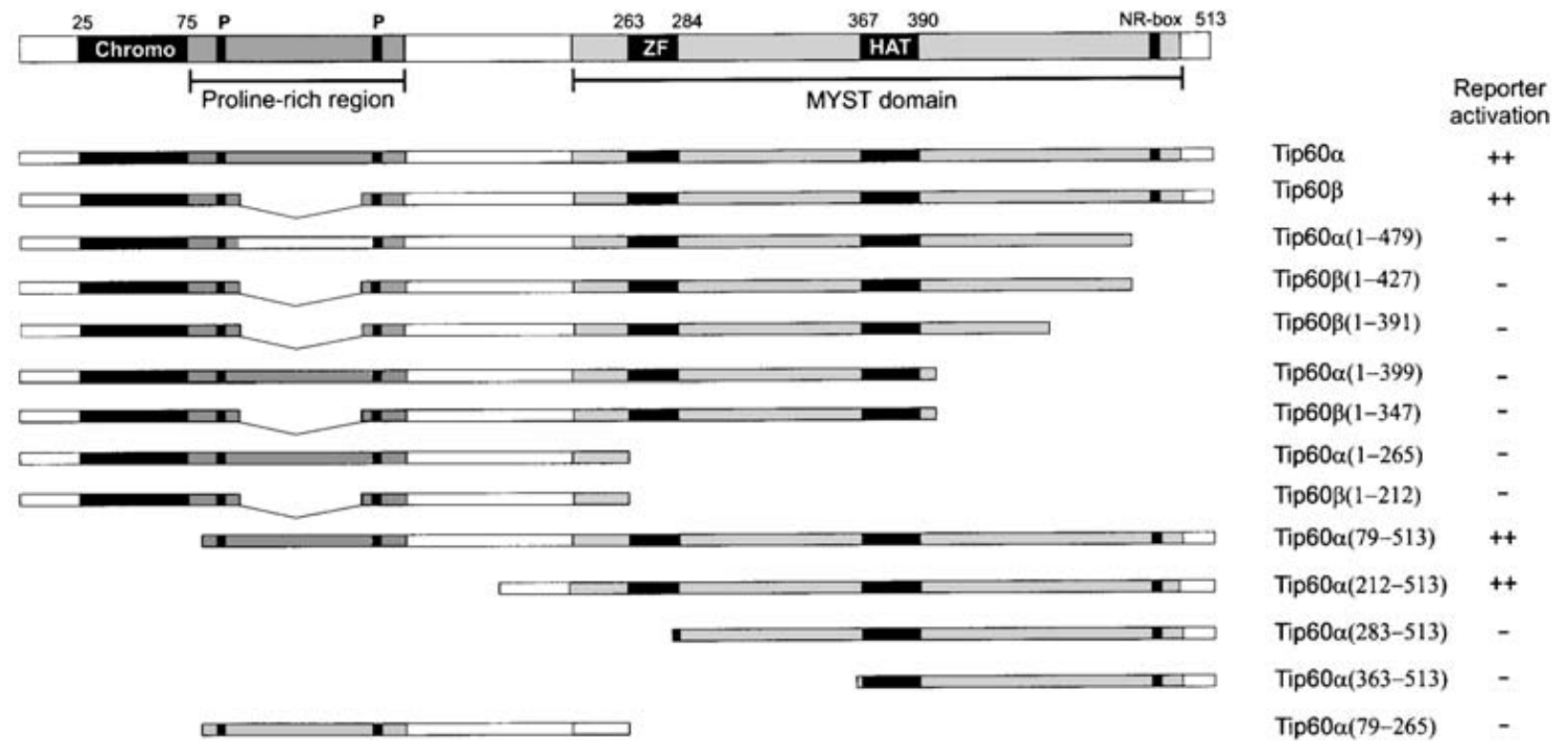

\section{Figure 3 TEL interaction region in Tip60}

TEL fused to the DBD LexA (1-202) and Tip60 deletions fused to the Gal4 (768-881) activation domain were tested for interaction in the yeast two-hybrid system using the yeast strain L40, containing the HIS3 and LacZ reporter genes. Interactions were detected as prototrophic growth on solid media lacking histidine and blue colouring when grown in the presence of X-Gal (see the Materials and methods section). The functional expression of the different Tip60 deletions was verified in the yeast two-hybrid system using other Tip60-interaction partners, such as BCL3 (obtained from Christian Langediil Christiansen, Department of Molecular Biology, Aarhus University, Denmark) or internal fragments of TEL (results not shown). C-terminal MYST in Tip60 is essential for TEL binding. P, potential extracellular-signal-regulated kinase 1/2 phosphorylation sites; ZF, zinc fingers. 
211 amino acids of Tip60 $\alpha$ [Tip60 $\alpha$ (212-513)], containing CHROMO-like domain and proline-rich region, were not involved in TEL binding. Deletion of the first part of the MYST domain including the $\mathrm{C}_{2} \mathrm{HC}$ zinc finger destroyed interaction with TEL [see Tip60 $\alpha$ (283-513) in Figure 3]. This suggests an important role for the zinc finger motif; however, the zinc finger alone is not responsible for TEL interaction, because the C-terminal deletions Tip60 $\alpha$ (1-479), Tip60 $\beta$ (1-427) and Tip60 $\beta$ (1-391), all containing both the zinc finger and the HAT domain, did not bind to TEL. The crystal structure of Esa1, the yeast orthologue of Tip60, shows that the $\beta 3$-turn- $\beta 4-\alpha 1$-loop region corresponding to the Tip60 $\mathrm{C}_{2} \mathrm{HC}$ region constitutes an independent subdomain that is not intermingled with the core MYST domain [46]. Accordingly, deletion of the zinc finger in Tip60 $\alpha$ will probably not have a profound impact on the folding of the core MYST domain. This again supports the notion that the $\mathrm{C}_{2} \mathrm{HC}$ zinc finger region by itself is critical for TEL binding. Taken together, the mapping analysis showed that the regions with the zinc finger region and the extreme C-terminus harbouring the NR-box were important for TEL binding.

\section{Mutating the Tip60 MYST zinc finger eliminates interaction with TEL in yeast}

To assess the roles of the Tip60 $\mathrm{C}_{2} \mathrm{HC}$ zinc finger motif and the NR-box in interaction with TEL, we used site-directed mutagenesis to create mutations in either motif. In Tip60 $\beta$, we introduced a Cys $\rightarrow$ Ala substitution in the first cysteine residue of the zinc finger creating Tip60 $\beta_{\mathrm{C} 211 \mathrm{~A}}$. In Tip60 $\beta_{\mathrm{L} 440 \mathrm{~A}, \mathrm{~L} 441 \mathrm{~A}}$, we substituted the last two leucine residues of the NR-box (LKRLL) with alanine residues. Tip60 $\beta_{\mathrm{C} 211 \mathrm{~A}}$ and Tip60 $\beta_{\mathrm{L} 440 \mathrm{~A}, \mathrm{~L} 441 \mathrm{~A}}$ were tested for interaction with TEL in yeast (Figure 4). Mutation of the zinc finger eliminates interaction with TEL (Figure 4, plate position 2), whereas the NR-box mutation did not influence interaction (Figure 4, plate position 3 ) when compared with wildtype (wt) Tip60 $\beta$ (Figure 4, plate position 1). Plate positions 4,5 and 6 represent co-transformation of Tip60 $\beta_{\mathrm{L} 440 \mathrm{~A}, \mathrm{~L} 441 \mathrm{~A}}$, $\operatorname{Tip} 60 \beta_{\mathrm{C} 211 \mathrm{~A}}$ and Tip60 $\beta$ respectively with empty bait vector and serve as negative controls. This analysis shows that the Tip60 zinc finger motif is critical for the TEL interaction.

\section{Interaction of TEL with Tip60 in vivo}

To confirm interaction between TEL and Tip60 in vivo, a mammalian two-hybrid assay was applied. TEL and the N-terminal deletion TEL (117-452) (see Figure 2) were fused to the Gal4 DBD, and Tip60 $\beta$ was fused to the activation domain of the herpes simplex virus transcription activator VP16, all in relevant mammalian expression vectors. Pairs of TEL and Tip60 constructs were transfected into HT1080 cells along with the SEAP reporter (Figure 5A) and interactions were monitored by measuring SEAP activity (Figure 5B). By installing Gal4 and VP16 at the Nterminal ends of TEL and Tip60 respectively, the risk of interfering with the interaction between TEL and Tip60 should be minimized, because the yeast interaction mapping identified the $\mathrm{C}$-terminal segments of both TEL and Tip60 as being responsible for the interaction. In Gal4-TEL, TEL may function as a transcription repressor and thus obscure the analysis. We therefore included the TEL (117-452) construct in the analysis. In independent studies, TEL represses two times more than TEL (117-452) (results not shown). Figure 5(B) shows that the presence of Gal4-TEL and Gal4-TEL (117-452) recruited Tip60-VP16 to the promoter of the reporter and activated SEAP expression, thus demonstrating in vivo interaction between TEL and Tip60.
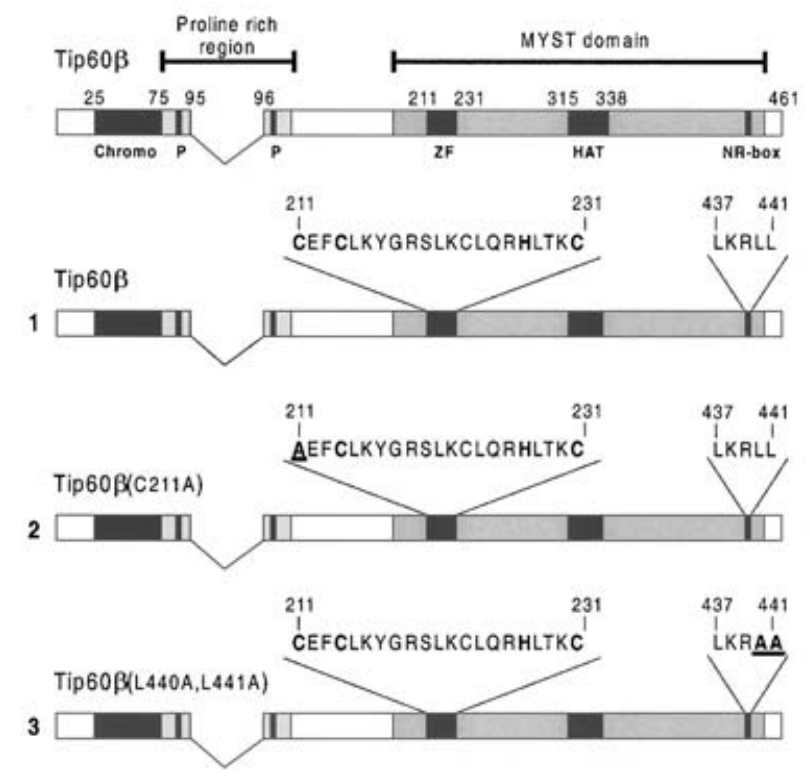

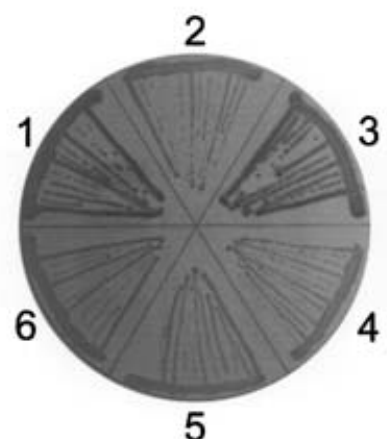

-TL plate + X-Gal overlay

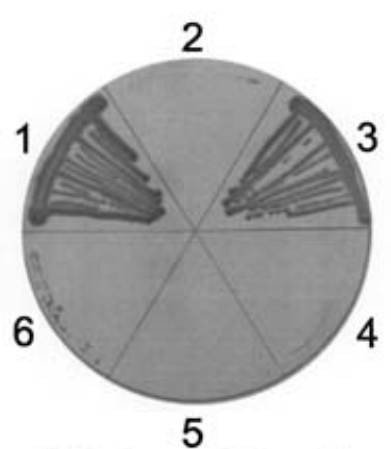

-TLH plate + X-Gal overlay
Figure 4 Zinc finger in the Tip60 MYST domain is essential for TEL interaction

TEL, fused to the DBD LexA (1-202), and Tip60 $\beta$ (1), Tip60 $\beta_{C 211 A}$ (2) and Tip60 $\beta_{\triangle 440 A, L 441 A}$ (3), fused to the Gal4 (768-881) activation domain were tested for interaction in the yeast strain $L 40$, containing the HIS3 and LacZ reporter genes. Interactions were detected as prototrophic growth on solid media lacking histidine residue and blue colouring when grown in the presence of $\mathrm{X}$-Gal (see the Materials and methods section). -TL and -TLH media plate positions 4, 5 and 6 are negative controls for Tip $60 \beta_{\triangle 440 A . \angle 441 A}$. Tip $60 \beta_{C 211 A}$ and Tip $60 \beta$ respectively, co-transformed with the empty bait vector pBTM116. Yeast colonies streaked on -TL plates were allowed to grow for 3 days and then transferred to -TLH plates and again grown for 3 days. -TL and -TLH media plates were then covered with X-Gal-containing agarose (see the Materials and methods section) and incubated for $24 \mathrm{~h}$ before pictures were taken.

It was shown in Figure 4 that the Tip60 zinc finger was critical for TEL interaction in yeast. To confirm this interaction in mammalian cells, we constructed VP16 fusions with Tip60 $\beta_{\mathrm{C} 211 \mathrm{~A}}$ and wt Tip60 $\beta$. These were tested against Gal4-TEL (117-452) using a SEAP reporter to measure the interaction (see Figure 5C). Co-transfection of Gal4-TEL (117-452) and VP16-Tip60 $\beta$ gave a 5-fold activation (lane 3). In contrast, co-transfection of Gal4TEL (117-452) and VP16-Tip60 $\beta_{\mathrm{C} 211 \mathrm{~A}}$ gave no activation (lane 4). Results showed unambiguously that the Tip60 $\mathrm{C}_{2} \mathrm{HC}$ zinc finger motif was essential for TEL binding in mammalian cells.

\section{Tip60 is a co-repressor in TEL-mediated transcription repression}

To characterize the effect of Tip60 binding to TEL on the transcription-regulatory properties, we constructed a TEL-responsive 

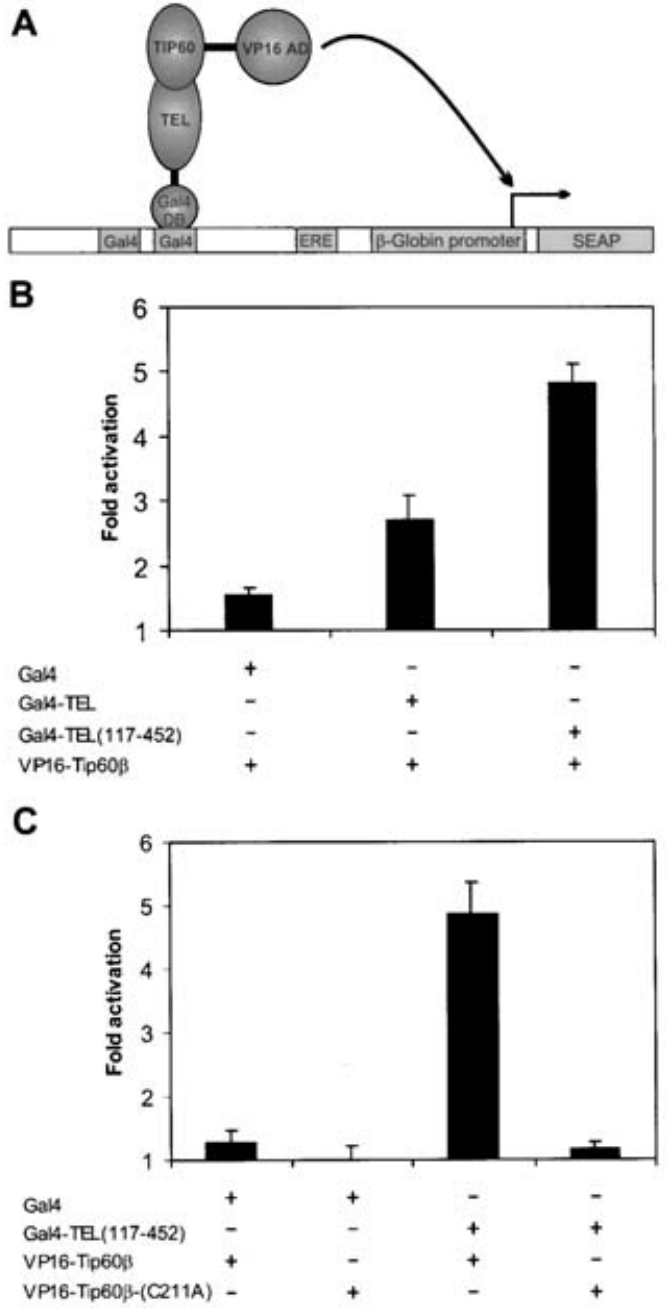

Figure 5 TEL and Tip60 interactions in vivo

(A) The mammalian two-hybrid system used for detection of the interactions between TEL and Tip60. The SEAP reporter is depicted. (B, C) Human fibroblast HT1080 cells were transfected with $1 \mu \mathrm{g}$ of SEAP reporter plasmid and $1 \mu \mathrm{g}$ of pCH110 $\beta$-gal plasmid (internal control of transfection), together with $1 \mu \mathrm{g}$ of each construct listed under the columns. Gal4, Gal4 alone; Gal4-TEL, Gal4 fused to full-length TEL; Gal4-TEL (117-452), Gal4 fused to TEL (amino acids 117-452); VP16-Tip60 $\beta$, VP16 fused to full-length Tip60 $\beta$; VP16-Tip60 $\beta$ (C211A), VP16 fused to Tip60 $\beta$ carrying a point mutation at position 211. Fold activation is calculated as VP16-Tip60 $\beta$ NP16 and VP16-Tip60 $\beta_{C 211 A}$ NP16 after normalization to the internal $\beta$-gal reporter. All transfections were performed in triplicate and SEAP activity/h was normalized to the value of the empty plasmid that is set to 1 (= no activation).

reporter. The ETS domain of the ETS transcription factors recognizes specific DNA sequences termed as EBS. These are composed of a core GGA trinucleotide sequence and their ETSbinding specificity is governed by short sequences (nt 3-5) flanking the GGA sequence. The TEL EBS consensus sequence is reported to be TG/TA/CGGAAGT [13] and the sequence ATAAACAGGAAGTGG is also shown to bind TEL [2]. Three different TEL EBS sequences (TTCGGAAGT, TGAGGAAGT and ATAAACAGGAAGTGG) were inserted side by side into the SEAP reporter at the enhancer position next to the oestrogen receptor (ER)-binding site, creating the TEL-EBS reporter (Figure 6A). HT1080 cells were transfected with TEL and Tip60 expression vectors, the TEL-EBS reporter and finally plasmids expressing transcription activators Gal4-E1A (Figure 6B) or ERVP16 (Figure 6C). The effect on transcription was estimated by
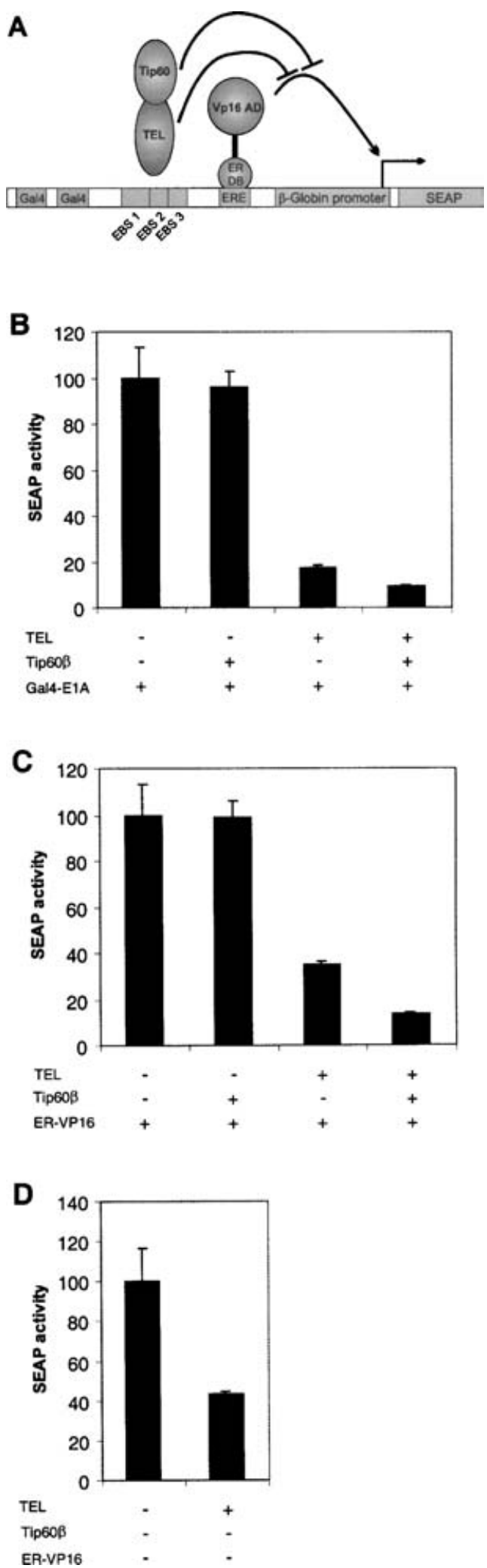

Figure 6 Tip60 is a co-repressor for TEL

(A) Modified SEAP reporter system. The EBS-SEAP reporter carries binding sites for TEL. (B-D) HT1080 cells were transfected with $0.5 \mu \mathrm{g}$ of each of the construct listed under the columns ( + , with insert; - , corresponding plasmid without insert), $1 \mu \mathrm{g}$ of EBS-SEAP reporter plasmid and $25 \mathrm{ng}$ of ER-VP16 or ER-E1A to enhance transcription. All transfections were performed in triplicate and SEAP activity/h was normalized to the value of the empty plasmids that is set to 100 . 
measuring SEAP activity. Tip60 had no effect on the reporter (columns 2) and TEL represses the reporter transcription by approx. 3-fold (columns 3), which is comparable with Gal4TEL repression of the SEAP reporter without the TEL-binding sites (results not shown). Co-expression of Tip60 further represses the transcription in a TEL-dependent manner (columns 4). Figure 6(D) shows that expression of TEL without overexpression of transcription activators still gave a significant repression, confirming that TEL mediates active repression and not passive repression by blocking ER-VP16 or Gal4-E1A binding. We conclude that Tip60 functionally attenuates TEL-EBS reporter expression in a TEL-dependent manner.

\section{DISCUSSION}

The first interest in TEL arose from its involvement in chromosomal translocations associated with haematological malignancies. At present, TEL is known to be involved in more than 40 translocations and the most frequent, the $\mathrm{t}(12 ; 21)(\mathrm{p} 13 ; \mathrm{q} 22)$, fuses the TEL gene to the AML1 transcription activator. This translocation is associated with B-cell ALL, present in 25-30\% of paediatric and $3 \%$ of adult B-cell ALL [14,47]. TEL belongs to the large and rapidly growing family of ETS transcription factors. The ETS protein family encompasses more than 45 members, each containing the highly conserved ETS DBD of approx. 85 amino acids. The ETS domain binds to purine-rich DNA sequences containing the core-binding motif GGAA/T, which is flanked on both sides by $2-4$ nucleotides giving specificity [13]. ETS proteins bind to unique EBS either alone or in combination with other assisting proteins. ETS transcription-factor-regulated genes are involved in numerous cellular processes, including proliferation, differentiation, migration/tissue remodelling, haematopoiesis, angiogenesis, apoptosis and cell transformation [48-52]. TEL is essential for yolk sac angiogenesis and for survival of neural and mesenchymal cells [3]. Further analysis of haematopoietic progenitor cells of all lineages from chimaeric mice generated by transfer of $\mathrm{TEL}^{-/-} \mathrm{ES}$ cells to blastocysts revealed that TEL was dispensable for adult-type haematopoietic lineages in the yolk sac and foetal liver, but is required specifically for establishment of bone-marrow haematopoiesis of all lineages [53].

In the present study, we have shown that TEL interacts directly with the MYST HAT protein Tip60. The interaction region of TEL is located within the $200 \mathrm{C}$-terminal amino acids that include the ETS domain. We note that regions outside the classical ETS domain seem to be involved in Tip60 binding, but we cannot exclude that these regions may affect the overall conformation of TEL. The TEL interaction domain of Tip60 was mapped to a region including the $300 \mathrm{C}$-terminal amino acids with the MYST domain. From analysis of the C-terminal deletions, we observed that deletion of the amino acids 212-283 abrogated interactions with TEL. This segment includes the C-terminal end of the MYST domain and, thus, we cannot exclude that it may affect the overall structure of the MYST domain. The MYST domain includes three distinct structures: a zinc finger, a HAT domain and an NRbinding domain (see Figure 3). The crystal structure of the yeast MYST protein, Esa1, shows that both the zinc finger and the NR corresponding domain are located at the surface of the protein. As Tip60 is homologous with Esa1, we tested whether these two domains were involved in TEL interactions, and we found in yeast and mammalian two-hybrid experiments that the $\mathrm{C}_{2} \mathrm{HC}$ zinc finger motif is essential for TEL interaction, whereas the Cterminal NR-box was found to be dispensable. The observation that the DBD in TEL is involved in the Tip60 interaction raised the question of whether DNA-binding ability of TEL was affected by Tip60.

We found that Tip60 attenuated transcription in a TELdependent manner and, thus, performed functionally as a corepressor. A co-repressor function may be achieved by recruiting other repressors as histone-modifying enzymes. Alternatively, repression may be completed by stabilization of the repressor or its binding to DNA. Hence, we have shown that Tip60 and TEL interacted in yeast and mammalian cells in vivo. The most probable explanation is that the Tip60 effect may be effectuated by a direct interaction with TEL, presumably in a complex of proteins instituted at the promoter. However, we cannot exclude an indirect effect of Tip60 that stimulates the expression or the function of another factor that supports Tel repression. Tip60 is a co-activator of the androgen receptor (AR). Tip60 acetylates AR, and HAT mutations eliminate Tip60-mediated co-activation of AR [34]. Thus we have analysed the acetylation potential of recombinant Tip60 in vitro and found that Tip60, in addition to being autoacetylated, acetylated histone $\mathrm{H} 3$ and BSA, but recombinant TEL was not a substrate for Tip60 acetylation (results not shown) suggesting that DNA-binding ability of TEL is not directly modified by acetylation. However, as noted, Tip60 may stimulate the interaction between TEL and its co-repressors mSin3A, silencing mediator for retinoid and thyroid receptor (SMRT)/ nuclear co-repressor (NCoR) and HDAC-3 [19-21,54]. Bandshift and in vivo functional analysis of TEL indicate that in full-length TEL, the ETS region may be masked/inhibited intramolecularly [18]. We also note that, in TEL, the C-terminal ETS-flanking region is composed of acidic amino acid that may interfere with DNA binding. On Tip60 binding, a conformational change might expose the ETS domain or inhibitory regions may be shielded. If the weak TEL DNA-binding affinity is caused by such intramolecular inhibition of the ETS domain, Tip60 binding might remove this inhibition and expose the ETS motif. Studies of the eukaryotic ETS transcription factor, Ets1, showed that the DNA binding is regulated negatively by intermolecular inhibition up to 23 -fold and that two regions flanking the ETS domain mediate the inhibition by promoting the dissociation from DNA $[55,56]$. Interestingly, in the transcription factor Ets 1 , the $\mathrm{N}$ - and $\mathrm{C}$-flanking inhibitory regions function in an inter-dependent mode. Concerning TEL, it is probable that $\mathrm{N}$ - and $\mathrm{C}$-terminal regions of the ETS domain interact, mask the ETS domain and form an interaction interface to Tip60. The Drosophila MYST protein MOF (Tip60 homologue) uses its $\mathrm{C}_{2} \mathrm{HC}$ zinc finger to bind the globular part of the nucleosome and the tail of histone $\mathrm{H} 4$ [57]. Accordingly, it is possible that the Tip60 $\mathrm{C}_{2} \mathrm{HC}$ zinc finger binds TEL and assists in DNA-binding/complex formation.

As noted, Tip60 is important for DNA repair and apoptotic signalling. If the Tip60 complex requires interaction with TEL for these processes, this could in association with the observed loss of the non-translocated TEL allele in $\mathrm{t}(12 ; 21)(\mathrm{p} 13 ; \mathrm{q} 22)-$ associated ALL explain progression in the malignancy. The Band T-cells are vulnerable especially to genetic lesions as B- and T-cells exhibit high activity of immunoglobulin and T-cell receptor gene rearrangements. As TEL is expressed highly in the haematopoietic cells, it may, in complex with Tip60, play a pivotal role in protecting the cell from mutations; in contrast, when absent from ALL cells, TEL may lead to accumulation of new putative oncogenic mutations.

We thank Claus Bus for technical assistance, and A. L. Nielsen and C. L. Christiansen for the donation of plasmids. We further thank B. J. Bonven and A. L. Nielsen for fruitful discussions during the planning and interpretation of the analysis. This work was supported by research grants from the Danish Cancer Society, The Danish Natural Science Council and Inge Elise Jensen Foundation. 


\section{REFERENCES}

1 Golub, T. R., Barker, G. F., Lovett, M. and Gilliland, D. G. (1994) Fusion of PDGF receptor $\beta$ to a novel ets-like gene, tel, in chronic myelomonocytic leukemia with $\mathrm{t}(5 ; 12)$ chromosomal translocation. Cell (Cambridge, Mass.) 77, 307-316

2 Poirel, H., Oury, C., Carron, C., Duprez, E., Laabi, Y., Tsapis, A., Romana, S. P., Mauchauffe, M., Le Coniat, M., Berger, R. et al. (1997) The TEL gene products: nuclear phosphoproteins with DNA binding properties. Oncogene 14, 349-357

3 Wang, L. C., Kuo, F., Fujiwara, Y., Gilliland, D. G., Golub, T. R. and Orkin, S. H. (1997) Yolk sac angiogenic defect and intra-embryonic apoptosis in mice lacking the Ets-related factor TEL. EMBO J. 16, 4374-4383

4 Golub, T. R., Goga, A., Barker, G. F., Afar, D. E., McLaughlin, J., Bohlander, S. K., Rowley, J. D., Witte, O. N. and Gilliland, D. G. (1996) Oligomerization of the ABL tyrosine kinase by the Ets protein TEL in human leukemia. Mol. Cell. Biol. 16, 4107-4116

5 lijima, Y., Ito, T., Oikawa, T., Eguchi, M., Eguchi-Ishimae, M., Kamada, N., Kishi, K., Asano, S., Sakaki, Y. and Sato, Y. (2000) A new ETV6/TEL partner gene, ARG (ABL-related gene or ABL2), identified in an AML-M3 cell line with a t(1;12)(q25;p13) translocation. Blood 95, 2126-2131

6 Lacronique, V., Boureux, A., Valle, V. D., Poirel, H., Quang. C. T., Mauchauffe, M., Berthou, C., Lessard, M., Berger, R., Ghysdael, J. et al. (1997) A TEL-JAK2 fusion protein with constitutive kinase activity in human leukemia. Science 278, 1309-1312

7 Eguchi, M., Eguchi-Ishimae, M., Tojo, A., Morishita, K., Suzuki, K., Sato, Y., Kudoh, S. Tanaka, K., Setoyama, M., Nagamura, F. et al. (1999) Fusion of ETV6 to neurotrophin-3 receptor TRKC in acute myeloid leukemia with t(12;15)(p13;q25). Blood 93 1355-1363

8 Andersen, M. T., Nordentoft, I., Hjalgrim, L. L., Christiansen, C. L., Jakobsen, V. D. Hjalgrim, H., Pallisgaard, N., Madsen, H. O., Christiansen, M., Ryder, L. P. et al. (2001) Characterization of $\mathrm{t}(12 ; 21)$ breakpoint junctions in acute lymphoblastic leukemia. Leukemia 15, 858-859

9 Golub, T. R., Barker, G. F., Bohlander, S. K., Hiebert, S. W., Ward, D. C., Bray-Ward, P., Morgan, E., Raimondi, S. C., Rowley, J. D. and Gilliland, D. G. (1995) Fusion of the TEL gene on 12p13 to the AML1 gene on 21q22 in acute lymphoblastic leukemia. Proc. Natl. Acad. Sci. U.S.A. 92, 4917-4921

10 Romana, S. P., Poirel, H., Leconiat, M., Flexor, M. A., Mauchauffe, M., Jonveaux, P., Macintyre, E. A., Berger, R. and Bernard, O. A. (1995) High frequency of t(12;21) in childhood B-lineage acute lymphoblastic leukemia. Blood 86, 4263-4269

11 Peeters, P., Wlodarska, I., Baens, M., Criel, A., Selleslag, D., Hagemeijer, A., Van den, B. H. and Marynen, P. (1997) Fusion of ETV6 to MDS1/EVI1 as a result of $\mathrm{t}(3 ; 12)(\mathrm{q} 26 ; \mathrm{p} 13)$ in myeloproliferative disorders. Cancer Res. 57, 564-569

12 Chase, A., Reiter, A., Burci, L., Cazzaniga, G., Biondi, A., Pickard, J., Roberts, I. A., Goldman, J. M. and Cross, N. C. (1999) Fusion of ETV6 to the caudal-related homeobox gene CDX2 in acute myeloid leukemia with the $t(12 ; 13)(p 13 ; q 12)$. Blood $93,1025-1031$

13 Szymczyna, B. R. and Arrowsmith, C. H. (2000) DNA binding specificity studies of four ETS proteins support an indirect read-out mechanism of protein-DNA recognition. J. Biol. Chem. 275, 28363-28370

14 Shurtleff, S. A., Buijs, A., Behm, F. G., Rubnitz, J. E., Raimondi, S. C., Hancock, M. L., Chan, G. C., Pui, C. H., Grosveld, G. and Downing, J. R. (1995) TEL/AML1 fusion resulting from a cryptic $\mathrm{t}(12 ; 21)$ is the most common genetic lesion in pediatric $\mathrm{ALL}$ and defines a subgroup of patients with an excellent prognosis. Leukemia 9, 1985-1989

15 Cave, H., Cacheux, V., Raynaud, S., Brunie, G., Bakkus, M., Cochaux, P., Preudhomme, C., Lai, J. L., Vilmer, E. and Grandchamp, B. (1997) ETV6 is the target of chromosome $12 p$ deletions in $\mathrm{t}(12 ; 21)$ childhood acute lymphocytic leukemia. Leukemia 11. $1459-1464$

16 Fenrick, R., Wang, L., Nip, J., Amann, J. M., Rooney, R. J., Walker-Daniels, J., Crawford, H. C., Hulboy, D. L., Kinch, M. S., Matrisian, L. M. et al. (2000) TEL, a putative tumor suppressor, modulates cell growth and cell morphology of ras-transformed cells while repressing the transcription of stromelysin-1. Mol. Cell. Biol. 20, 5828-5839

17 Van Rompaey, L., Holland, E. and Grosveld, G. (2002) TEL induces aggregation in transformed cells and induces tube formation in NIH3T3-UCLA cells. Biochem. Biophys. Res. Commun. 291, 820-828

18 Lopez, R. G., Carron, C., Oury, C., Gardellin, P., Bernard, O. and Ghysdael, J. (1999) TEL is a sequence-specific transcriptional repressor. J. Biol. Chem. 274, 30132-30138

19 Chakrabarti, S. R. and Nucifora, G. (1999) The leukemia-associated gene TEL encodes a transcription repressor which associates with SMRT and mSin3A. Biochem. Biophys. Res. Commun. 264, 871-877

20 Fenrick, R., Amann, J. M., Lutterbach, B., Wang, L., Westendorf, J. J., Downing, J. R. and Hiebert, S. W. (1999) Both TEL and AML-1 contribute repression domains to the t(12;21) fusion protein. Mol. Cell. Biol. 19, 6566-6574

21 Guidez, F., Petrie, K., Ford, A. M., Lu, H., Bennett, C. A., MacGregor, A., Hannemann, J., Ito, Y., Ghysdael, J., Greaves, M. et al. (2000) Recruitment of the nuclear receptor corepressor N-CoR by the TEL moiety of the childhood leukemia-associated TEL-AML1 oncoprotein. Blood 96, 2557-2561
22 Kamine, J., Elangovan, B., Subramanian, T., Coleman, D. and Chinnadurai, G. (1996) Identification of a cellular protein that specifically interacts with the essential cysteine region of the HIV-1 Tat transactivator. Virology 216, 357-366

23 Sterner, D. E. and Berger, S. L. (2000) Acetylation of histones and transcription-related factors. Microbiol. Mol. Biol. Rev. 64, 435-459

24 Jacobson, R. H., Ladurner, A. G., King, D. S. and Tjian, R. (2000) Structure and function of a human TAFII250 double bromo domain module. Science 288, 1422-1425

25 Sewack, G. F., Ellis, T. W. and Hansen, U. (2001) Binding of TATA binding protein to a naturally positioned nucleosome is facilitated by histone acetylation. Mol. Cell. Biol. 21 1404-1415

26 Kimura, A. and Horikoshi, M. (1998) Tip60 acetylates six lysines of a specific class in core histones in vitro. Genes Cells 3, 789-800

27 Yamamoto, T. and Horikoshi, M. (1997) Novel substrate specificity of the histone acetyltransferase activity of HIV-1-Tat interactive protein Tip60. J. Biol. Chem. 272 30595-30598

28 Ikura, T., Ogryzko, V. V., Grigoriev, M., Groisman, R., Wang, J., Horikoshi, M., Scully, R., Qin, J. and Nakatani, Y. (2000) Involvement of the TIP60 histone acetylase complex in DNA repair and apoptosis. Cell (Cambridge, Mass.) 102, 463-473

29 Smith, E. R., Eisen, A., Gu, W., Sattah, M., Pannuti, A., Zhou, J., Cook, R. G., Lucchesi, J. C. and Allis, C. D. (1998) ESA1 is a histone acetyltransferase that is essential for growth in yeast. Proc. Natl. Acad. Sci. U.S.A. 95, 3561-3565

30 Kinoshita, A., Whelan, C. M., Berezovska, 0. and Hyman, B. T. (2002) The $\gamma$ secretase-generated carboxyl-terminal domain of the amyloid precursor protein induces apoptosis via Tip60 in $\mathrm{H} 4$ cells. J. Biol. Chem. 277, 28530-28536

31 Legube, G., Linares, L. K., Lemercier, C., Scheffner, M., Khochbin, S. and Trouche, D. (2002) Tip60 is targeted to proteasome-mediated degradation by Mdm2 and accumulates after UV irradiation. EMBO J. 21, 1704-1712

32 Brady, M. E., Ozanne, D. M., Gaughan, L., Waite, I., Cook, S., Neal, D. E. and Robson, C. N. (1999) Tip60 is a nuclear hormone receptor coactivator. J. Biol. Chem. 274, 17599-17604

33 Gaughan, L., Brady, M. E., Cook, S., Neal, D. E. and Robson, C. N. (2001) Tip60 is a co-activator specific for class I nuclear hormone receptors. J. Biol. Chem. 276 $46841-46848$

34 Gaughan, L., Logan, I. R., Cook, S., Neal, D. E. and Robson, C. N. (2002) Tip60 and histone deacetylase 1 regulate androgen receptor activity through changes to the acetylation status of the receptor. J. Biol. Chem. 277, 25904-25913

35 Lyngso, C., Bouteiller, G., Damgaard, C. K., Ryom, D., Sanchez-Munoz, S., Norby, P. L., Bonven, B. J. and Jørgensen, P. (2000) Interaction between the transcription factor SPBP and the positive cofactor RNF4. An interplay between protein binding zinc fingers. J. Biol. Chem. 275, 26144-26149

36 Fields, S. and Song, 0. (1989) A novel genetic system to detect protein-protein interactions. Nature (London) 340, 245-246

37 Chakrabarti, S. R., Sood, R., Ganguly, S., Bohlander, S., Shen, Z. and Nucifora, G. (1999) Modulation of TEL transcription activity by interaction with the ubiquitin-conjugating enzyme UBC9. Proc. Natl. Acad. Sci. U.S.A. 96, 7467-7472

38 Liu, B., Liao, J., Rao, X., Kushner, S. A., Chung, C. D., Chang, D. D. and Shuai, K. (1998) Inhibition of Stat1-mediated gene activation by PIAS1. Proc. Natl. Acad. Sci. U.S.A. $\mathbf{9 5}$ 10626-10631

39 Imai, Y., Kimura, T., Murakami, A., Yajima, N., Sakamaki, K. and Yonehara, S. (1999) The CED-4-homologous protein FLASH is involved in Fas-mediated activation of caspase-8 during apoptosis. Nature (London) 398, 777-785

40 Kiriakidou, M., Driscoll, D. A., Lopez-Guisa, J. M. and Strauss, III, J. F. (1997) Cloning and expression of primate Daxx cDNAs and mapping of the human gene to chromosome $6 \mathrm{p} 21.3$ in the MHC region. DNA Cell Biol. 16, 1289-1298

41 Li, R., Pei, H., Watson, D. K. and Papas, T. S. (2000) EAP1/Daxx interacts with ETS1 and represses transcriptional activation of ETS1 target genes. Oncogene 19,745-753

42 Still, I. H. and Cowell, J. K. (1998) The t(8;13) atypical myeloproliferative disorder: further analysis of the ZNF198 gene and lack of evidence for multiple genes disrupted on chromosome 13. Blood 92, 1456-1458

43 Sohal, J., Reiter, A., Goldman, J. M. and Cross, N. C. (2000) Cloning of ZNF237, a novel member of the MYM gene family that maps to human chromosome 13q11-q12. Cytogenet. Cell Genet. 89, 24-28

44 Lu, K. P., Hanes, S. D. and Hunter, T. (1996) A human peptidyl-prolyl isomerase essential for regulation of mitosis. Nature (London) 380, 544-547

45 Yaffe, M. B., Schutkowski, M., Shen, M., Zhou, X. Z., Stukenberg, P. T., Rahfeld, J. U., Xu, J., Kuang, J., Kirschner, M. W., Fischer, G. et al. (1997) Sequence-specific and phosphorylation-dependent proline isomerization: a potential mitotic regulatory mechanism. Science 278, 1957-1960

46 Yan, Y., Barlev, N. A., Haley, R. H., Berger, S. L. and Marmorstein, R. (2000) Crystal structure of yeast Esa1 suggests a unified mechanism for catalysis and substrate binding by histone acetyltransferases. Mol. Cell 6, 1195-1205 
47 Ford, A. M., Bennett, C. A., Price, C. M., Bruin, M. C., Van Wering, E. R. and Greaves, M. (1998) Fetal origins of the TEL-AML1 fusion gene in identical twins with leukemia. Proc. Natl. Acad. Sci. U.S.A. 95, 4584-4588

48 Bartel, F. O., Higuchi, T. and Spyropoulos, D. D. (2000) Mouse models in the study of the Ets family of transcription factors. Oncogene $\mathbf{1 9}, 6443-6454$

$49 \mathrm{Hsu}$, T. and Schulz, R. A. (2000) Sequence and functional properties of Ets genes in the model organism Drosophila. Oncogene 19,6409-6416

50 Maroulakou, I. G. and Bowe, D. B. (2000) Expression and function of Ets transcription factors in mammalian development: a regulatory network. Oncogene 19, 6432-6442

51 Sharrocks, A. D. (2001) The ETS-domain transcription factor family. Nat. Rev. Mol. Cell Biol. 2, 827-837

52 Wasylyk, B., Hagman, J. and Gutierrez-Hartmann, A. (1998) Ets transcription factors: nuclear effectors of the Ras-MAP-kinase signaling pathway. Trends Biochem. Sci. 23, $213-216$

Received 8 January 2003/7 May 2003; accepted 9 May 2003

Published as BJ Immediate Publication 9 May 2003, DOI 10.1042/BJ20030087
53 Wang, L. C., Swat, W., Fujiwara, Y., Davidson, L., Visvader, J., Kuo, F., Alt, F. W. Gilliland, D. G., Golub, T. R. and Orkin, S. H. (1998) The TEL/ETV6 gene is required specifically for hematopoiesis in the bone marrow. Genes Dev. 12, 2392-2402

54 Wang, H., Huang, Z. Q., Xia, L., Feng, Q., Erdjument-Bromage, H., Strahl, B. D., Briggs, S. D., Allis, C. D., Wong, J., Tempst, P. et al. (2001) Methylation of histone $\mathrm{H} 4$ at arginine 3 facilitating transcriptional activation by nuclear hormone receptor. Science $\mathbf{2 9 3}$ 853-857

55 Garvie, C. W., Pufall, M. A., Graves, B. J. and Wolberger, C. (2002) Structural analysis of the autoinhibition of Ets-1 and its role in protein partnerships. J. Biol. Chem. $\mathbf{2 7 7 ,}$ 45529-45536

56 Jonsen, M. D., Petersen, J. M., Xu, Q. P. and Graves, B. J. (1996) Characterization of the cooperative function of inhibitory sequences in Ets-1. Mol. Cell. Biol. 16, 2065-2073

57 Akhtar, A. and Becker, P. B. (2001) The histone $\mathrm{H} 4$ acetyltransferase MOF uses a $\mathrm{C}_{2} \mathrm{HC}$ zinc finger for substrate recognition. EMBO Rep. 2, 113-118 